\title{
Combinatorial Problems in OpenAD
}

\author{
Jean Utke ${ }^{1}$ and Uwe Naumann ${ }^{2}$ \\ 1 Argonne National Laboratory, MCS \\ 9700 S. Cass Ave., Argonne, IL 60439, USA \\ utke@mcs.anl.gov \\ 2 RWTH Aachen University, LuFG Informatik 12 \\ 52056 Aachen, Germany \\ naumannestce.rwth-aachen.de
}

\begin{abstract}
Computing derivatives using automatic differentiation methods entails a variety of combinatorial problems. The OpenAD tool implements automatic differentiation as source transformation of a program that represents a numerical model. We select three combinatorial problems and discuss the solutions implemented in OpenAD. Our intention is to explain the specific parts of the implementation so that readers can easily use OpenAD to investigate and develop their own solutions to these problems.
\end{abstract}

Keywords. automatic differentiation, combinatorial problems, OpenAD

\section{Introduction}

Computing derivatives of numerical models $\boldsymbol{f}(\boldsymbol{x}) \mapsto \boldsymbol{y}: \mathbb{R}^{n} \mapsto \mathbb{R}^{m}$ given as a computer program $P$ is an important but also compute-intensive task. Automatic differentiation (AD) [1] provides the means to obtain such derivatives. OpenAD [2] implements $\mathrm{AD}$ as a source transformation applied to Fortran programs for both the forward and reverse modes. In this paper we describe the solutions to three combinatorial aspects of $\mathrm{AD}$ as implemented in OpenAD. For information regarding the general use of OpenAD please see [3]. Because OpenAD is a source transformation tool, the combinatorial problems are solved at compile time; hence, our approach can afford costly heuristics to approximate a good solution. Using such heuristics even with small improvements is justified when, for instance, these improvements benefit the numerical kernel of a loop and therefore the improvement accumulates over the runtime, realizing considerable savings. Our intention is to explain the representation of the combinatorial problems in OpenAD such that the reader will find it easy to implement and investigate other solutions to these problems. The remainder of this section provides a brief overview of the concepts of $\mathrm{AD}$ that are relevant to the problems in question. General information on $\mathrm{AD}$ can be found in recent conference proceedings [4,5], the AD community website [6], and the survey paper in this proceedings [7].

Automatic differentiation relies on the chain rule applied to a sequence of elemental operations. These operations are the function calls and intrinsics built into the given programming language, such as sin, cos, or the operators + and *. Typically, right-handside expressions of assignment statements form single-expression-use graphs [8], and 
under certain conditions a sequence of single-expression-use graphs corresponding to a sequence of assignment statements in a given program can be flattened into a directed acyclic graph [9]. This graph is called the computational graph $G$. An example is shown in Fig. 1. The nonminimal vertices of $G$ represent values computed as a result of a sin-

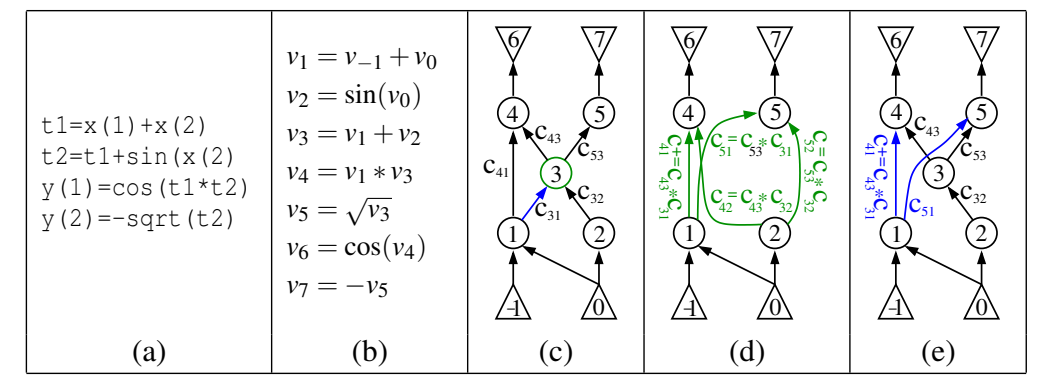

Fig. 1. Sequence of statements (a), corresponding sequence of elemental operations enumerating the computed values (b), the corresponding $G$ using the value enumeration (c), eliminaton of vertex 3 from $G(\mathrm{~d})$, and front elimination of edge $(1,3)$ from $G(\mathrm{e})$.

gle intrinsic function or operator call. For each such computed value $v_{i}$ we can provide the local partial derivative $c_{i j}=\partial v_{i} / \partial v_{j}$ with respect to its arguments $v_{j}$. For instance, in Fig. 1(b) we have $v_{4}=v_{1} * v_{3}$ and therefore $c_{41}=v_{3}$ and $c_{43}=v_{1}$. These partial derivatives are given as edge labels in $G$; see Fig. 1(c). Following Baur's formula [10], we can compute Jacobian entries by multiplying edge labels along paths from minimal to maximal edges in $G$ and adding parallel paths. Because of distributivity of the operations, the edge labels can be combined in many different orders yielding the same result but differing in the number of multiplication (and addition) operations performed. The multiplications and additions can be expressed as graph manipulations in $G$. Figure 1(d) shows a vertex elimination where the number of multiplications is equivalent to the Markowitz degree, and Figure 1(e) shows a front-edge elimination where the number of multiplications is equal to the number of out-edges of the target vertex; the modified elements are shown in color. For details and additional operations see [7,11] and [3], Sec. 3.2. Each elimination step modifies $G$ to some $G^{(k)}$. One can proceed with eliminations until the modified $G$ has reached bipartite form $G^{b}$ and the edge labels are the entries of the Jacobian $\boldsymbol{J}$ of $\boldsymbol{f}$ evaluated at some point $\boldsymbol{x}_{0}$. This description illustrates the basis of the following NP-complete [12] problem.

\section{What is the minimal number of multiplications to reduce $G$ to bipartite form?}

The OpenAD representation ${ }^{1}$ of the problem is discussed in Sec. 2.1 followed by example heuristics in Sec. 2.2.

\footnotetext{
${ }^{1}$ OpenAD is actively being developed. All names of files, classes, methods, and variables mentioned here refer to versions xaifBooster: b88d9f62ae82+, angel: ff7faed78ea6 of the respective Mercurial source code repositories (see [2]). Changes since then can be traced back by using the Mercurial web interface at http://mercurial.mcs.anl.gov/ad .
} 
In many practical applications one does not require the full $\boldsymbol{J}$ but only projections $\boldsymbol{J} \boldsymbol{S}$ or $\boldsymbol{W}^{T} \boldsymbol{J}$ with $\boldsymbol{S} \in \mathbb{R}^{n \times p}, \boldsymbol{W} \in \mathbb{R}^{m \times p}$, where $p$ is the number of desired directions. During the eliminations described above, the number of edges (with nonunit/nonconstant labels) at any intermediate stage $G^{(k)}$ often is smaller than that of the final $G^{b}$. Consequently, the projection operations executed with $G^{(k)}$ are cheaper than with $G^{b}$. The general concept is known as scarcity [13] and has been investigated for practical use in [14]. Figure 2 shows an example for a rank-1 update. The initial $G$ has only $3 n$ edges, of which $2 n$ are constant. If we eliminate the intermediate vertex $z$ (see also Fig. 1(d)), we have $n^{2}$ nonconstant edges. Consequently, the projections $\boldsymbol{J} \boldsymbol{S}$ or $\boldsymbol{W}^{T} \boldsymbol{J}$ require only $3 n p$ operations compared to $n^{2} p$. This scenario leads to the second combinatorial problem.

2. What is a minimal representation $J^{*}$ for $\boldsymbol{J}$ ?

In Sec. 2.3 we will explain the implementation of a greedy heuristic to approximate the minimal representation, with particular consideration given to the additional savings possible when one considers some edge labels to have unit value.

Because in OpenAD we have to build $G$ at compile time, the limitations of an automatic code analysis $^{2}$ to identify computed values (on the

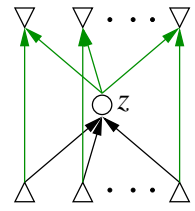

(a)

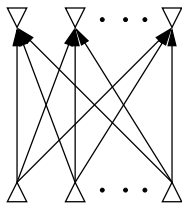

(b)

Fig. 2. Computational graph (edges with constant labels are green) for $\boldsymbol{f}(\boldsymbol{x})=\left(\boldsymbol{D}+\boldsymbol{a} \boldsymbol{x}^{T}\right) \boldsymbol{x}$ with an intermediate variable $z=\boldsymbol{x}^{T} \boldsymbol{x}$ in (a) and state after eliminating $z$ in (b). expressions) also limit the scope of any single $G$ typically to the contents of a basic block. Thus, for an entire program $P$ with control flow we have a sequence of $s$ graphs $G_{i}$ and, corresponding to that, a sequence of $s$ local Jacobians $\boldsymbol{J}_{i}$. The overall Jacobian $\boldsymbol{J} \in \mathbb{R}^{m \times n}$ for $P$ is therefore a chained matrix product over the $\boldsymbol{J}_{i}$. Again, instead of the full Jacobian $\boldsymbol{J}$ we typically require the projections $\left(\boldsymbol{J}_{S} \circ \ldots \circ\left(\boldsymbol{J}_{1} \circ \boldsymbol{S}\right) \ldots\right)$, called the forward mode, or $\left(\ldots\left(\boldsymbol{W}^{T} \circ \boldsymbol{J}_{S}\right) \circ \ldots \circ \boldsymbol{J}_{1}\right)$, called the reverse mode. Here we can also use the $\boldsymbol{J}_{i}^{*}$ in place of the less efficient $\boldsymbol{J}_{i}$. The bracketing for the reverse mode indicates that the $\boldsymbol{J}_{i}$ are required in an order inverse to the execution of the original program $P$. Typically, because of memory limitations, one will not be able to compute and store all the $\boldsymbol{J}_{i}$ at once to then use them for the reverse sweep. Instead one will have to trade off some recomputation of the $\boldsymbol{J}_{i}$ from checkpoints. Determining the checkpoints and orchestrating the computation of the $\boldsymbol{J}_{i}$ and their use in the reverse sweep is called a reversal scheme. This leads to the third combinatorial problem, also shown to be NPcomplete [15].

3. Which reversal scheme achieves the fewest recomputations, given constraints on the memory available for checkpoints and storing the $J_{i}$ ?

Section 3.1 highlights split and joint modes as two simple cases at the respective ends of the spectrum controlled at the level of subroutine calls. Section 3.3 discusses the optimal solution for the special case of uniform iterations.

\footnotetext{
2 This stems from aliasing, for example the possibility that two array elements a (i) and a ( $j$ ) or two pointers $\mathrm{p}$ and $\mathrm{q}$ point to the same address.
} 


\section{Computational Graphs}

The modular design of OpenAD separates different tasks of the source transformation. The core $\mathrm{AD}$ transformation engine is a module called xaifBooster separate from the modules for parsing, code analysis, and unparsing. An obvious candidate for the separation within the AD transformation engine is the computation of the local Jacobians $\boldsymbol{J}_{i}$ or $\boldsymbol{J}_{i}^{*}$, pertaining to problems 1 and 2 . We rely on the object-oriented language features of $\mathrm{C}++$ to facilitate the module separation via interface classes.

\subsection{Problem Representation}

To experiment with elimination heuristics, we do not need to understand how $G$ is constructed from code or how exactly the elimination steps are translated back into executable code. We need only an interface that describes the structure of $G$, the elimination steps, and (for problem 2) $G^{*}$, or remainder graph. The main interface is defined ${ }^{3}$ in the following file.

xaifBooster/algorithms/CrossCountryInterface/inc/Elimination.hpp

The structural representation of $G$ is a class called LinearizedComputationalGraph, or LCG. It uses vertex and edge classes LCGVertex and LCGEdge; their definitions can be found in the same directory in header files with the respective names. This graph class (like all other graph classes in xaifBooster) is based on the Boost Graph Library [16]. An edge elimination step such as the front elimination of edge $(1,3)$ shown in Fig. 1(e) yields the following two (because vertex 3 has two outedges) fused multiplyadd operations: $c_{41}{ }^{+}=c_{43}{ }^{\star} c_{31}$ and $c_{51}=c_{53}{ }^{\star} c_{31}$. They are represented as instances of the class JacobianAccumulationExpression, or JAE. Because we need only structural information, the nonminimal vertices of a $J A E$ represent the * or + operations, and the minimal vertices have references either to edges of $G$ or to maximal vertices of other $\mathrm{JAE}$ that are earlier elimination results, such as the new edge $(1,5)$ in Fig. 1(e). An instance of JAEList and the remainder graph $G^{*}$ (which, like the input graph $G$, is an instance of $L C G$ ) are the results of the top-level routine Elimination: : eliminate called from within xaifBooster.

Aside from the purely structural representation of $G$, the instances of LCGEdge contain a discriminator to identify whether an edge label is \pm 1 , constant, or generally variable. This information is needed to solve the second problem. In the following we will discuss elimination heuristics implemented by Angel [17,18].

\subsection{Elimination Heuristics}

The top-level driver routine for the heuristics is Elimination: :eliminate () implemented in angel/src/xaif_interface.cpp. An Elimination instance is instantiated from xaifBooster, and its attributes are set via oadDriver command line switches a,

\footnotetext{
${ }^{3}$ We follow the convention used in the OpenAD manual [3], Sec. 2.2, by referencing all files relative to the OpenAD install directory set as the \$OPENADROOT environment variable. We provide generated documentation of the source of all OpenAD components under the website's [2] "Documentation" link.
} 
A, m, M, and R; see [3], Sec. 4.1.3.4. The attributes determine which specific elimination routine is called by eliminate, for instance, compute_elimination_sequence (). ${ }^{4}$

Markowitz-Based Heuristics Using Markowitz (triggered by setting -M 0 ) as an example, we illustrate the implementation of a heuristic and identify in red the minimal set of elements to be changed for a new heuristic. Angel internally uses plain boost graphs. The first step in compute_elimination_sequence () is to convert the LCG given as input via a call to read_graph_xaif_booster. Then we declare a stack $\mathcal{F}$ of heuristics that filters the elimination target vertices down to a single vertex. For example, the first of three such $\mathcal{F}$ (for vertex elimination) is declared in xaif_interface.cpp, line 1151 , as

typedef heuristic_pair_t<lowest_markowitz_vertex_t>, reverse_mode...> lm_rm_t; and later, on line 1154 , is defined as follows.

lm_rm_t lm_rm_v (lowest_markowitz_vertex, reverse_mode_vertex);

This filter stack internally first passes vertices with the lowest Markowitz degree and then uses the reverse mode order as a tie breaker. The Markowitz filter and the re-

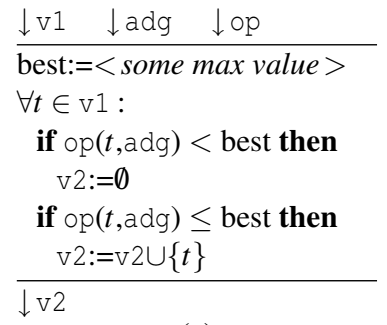

(a)

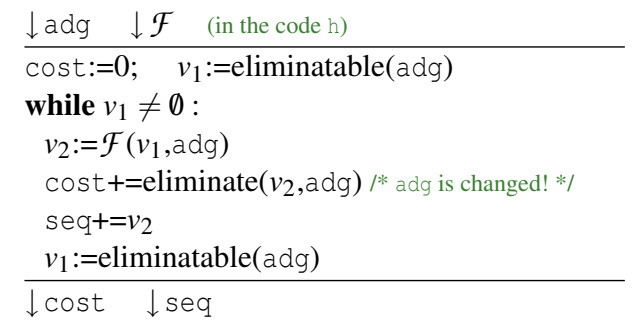

(b)

Fig. 3. Pseudo code for standard_heuristic_op (a) and for use_heuristic (b).

verse mode tie breaker are defined by using standard_heuristic_op and a function object ${ }^{5}$ called lmv_op_t, defined in angel/src/heuristics.cpp. Figure 3(a) shows the pseudo code implemented in standard_heuristic_op taking in a vector of elimination targets $\mathrm{v} 1$, a graph adg, and the aforementioned function object as a formal parameter op. The core of the Markowitz heuristic is encapsulated in the function object Imv_op_t's operator (), which returns the expected product of in and out degree.

$$
\text { in_degree }(\mathrm{v}, \mathrm{cg}) \text { * out_degree }(\mathrm{v}, \mathrm{cg})
$$

With this framework, the implementation of a new heuristic requires comparatively little effort, and one has the remainder of OpenAD readily available to evaluate its efficacy.

Evaluating the Cost Now that we have described how a filter stack is implemented, we return to compute_elimination_sequence () to look at the computation and comparison of the resulting cost. The above-mentioned three $\mathcal{F}$ together with simple forward and reverse order are passed in a call to best_heuristic (line 1169), which com-

\footnotetext{
${ }^{4}$ We encourage using the "search" provided in the Angel and xaifBooster generated source code documentation under "Documentation" at [2].

${ }^{5} \mathrm{C}++$ classes with an operator ().
} 
putes the elimination sequences and their respective cost individually and returns the cheapest elimination sequence. The definition is found in heuristics_impl.hpp. To determine the cost of each of the five $\mathcal{F}$ individually, it calls use_heuristic, defined in heuristics.cpp; see also Fig. 3(b). It takes as input the graph $G$ as adg and the filter and returns the cost and the elimination sequence seq. The filter $\mathcal{F}$ determines the next elimination target, and the cost is accumulated as incurred by the actual elimination. For the latter, one can follow the calls from eliminate to vertex_elimination to back_edge_elimination to see that the cost is the number of edge label multiplications. Consequently a new heuristic for this cost model does not necessitate any further changes.

Edge Eliminations Returning once more to compute_elimination_sequence (), we also find a variety of edge elimination heuristic filters declared. The logic employed for these is analogous to that applied to vertex eliminations. The cost, following the same cost model used for vertex elimination, for the best edge elimination is compared to that of the best vertex elimination. The winner is converted into an equivalent face elimination [11] sequence. Because a face elimination can be considered the elemental building block for all elimination operations, there is a common method to populate the caller-provided instance of JAEList which expects a sequence of face eliminations as input. An actual face elimination sequence and accompanying heuristics can be found in compute_elimination_sequence_lsa_face.

\subsection{Scarcity-Preserving Heuristics}

To approximate a minimal representation $\boldsymbol{J}^{*}$ of the Jacobian $\boldsymbol{J}$ (problem 2), OpenAD has various scarcity-preserving heuristics [14]. We describe a simple example of such a heuristic that is implemented in compute_partial_elimination_sequence. Its execution is triggered by the oadDriver command line settings $-\mathrm{M} 1[-\mathrm{m}]$. While the heuristics discussed in Sec. 2.2 minimize the count of elimination operations, the cost here is simply the number of nonunit edge labels in the remainder graph $G^{*}$. Minimizing the elimination-induced operations count is a secondary concern. Given the distinction of unit, constant, and variable edge labels in the LCGEdge class, we can disregard the multiplication of constant edge labels from the elimination operations cost because it is a compile time effort. All counters pertaining to the heuristic are kept in an instance of elimSeq_cost_t that is defined in angel/include/angel_types.hpp. The pseudo code in Fig. 4 illustrates the core logic. Here, for simplicity, we consider only edge elimination operations. Again we use a stack of filters to narrow the eligible targets down to a single edge. However, here we do so repeatedly because in each elimination sequence we may detect a refill ${ }^{6}$ that in a subsequent elimination sequence we attempt to avoid (line 09), thereby modifying the set $v_{3}$ and arriving at different result. After each elimination is complete, we compare it to the current best result, where elimseq_cost_t holds all relevant counters, such as the minimal edge count reached along the way, and the operations incurred. If no new refill dependence is detected, we are done. The easiest entry point to change the behavior for a new heuristic is to

\footnotetext{
${ }^{6}$ An edge $(i, j)$ is refilled if it is eliminated but subsequently recreated as a consequence of eliminating edges in an alternative path from $i$ to $j$.
} 


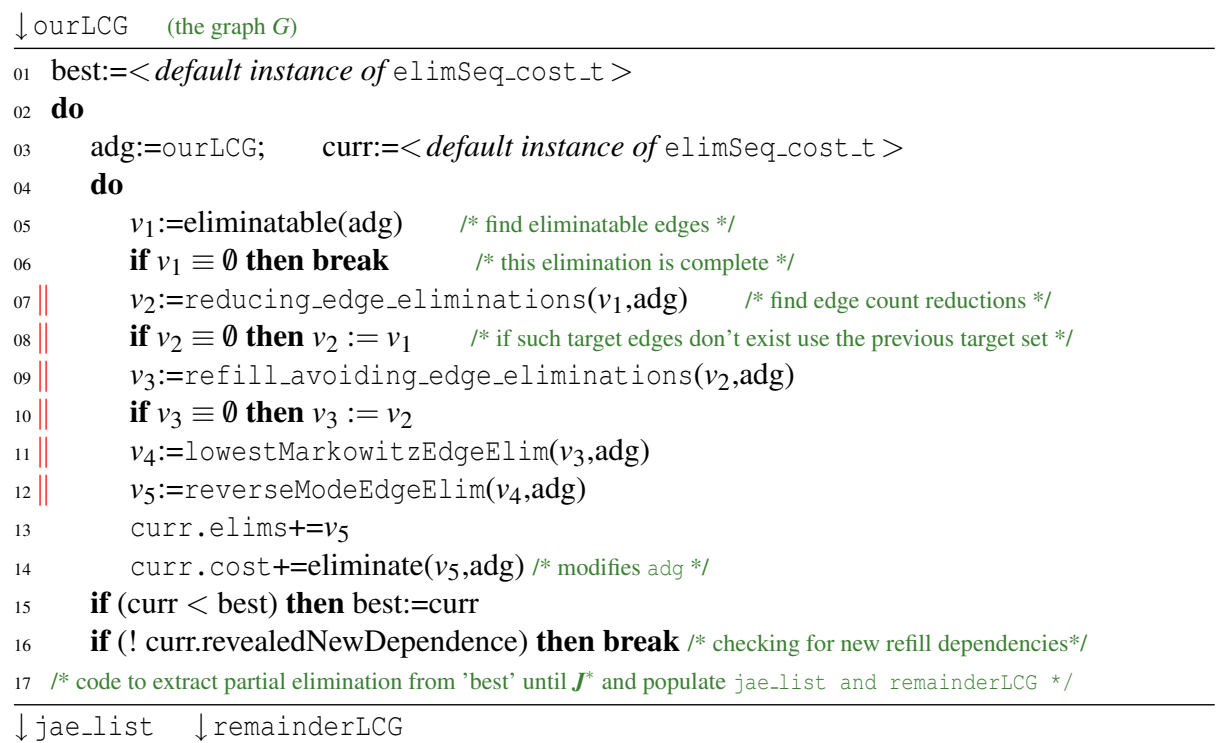

Fig. 4. Pseudo code for compute_partial_elimination_sequence

modify the filter stack at any of the red-marked lines $07-12$. To finish, we need to create $G^{*}$ by replaying the best elimination sequence to the first point when the minimal edge count is reached. This also populates the instance of JAEList. Finally we populate the caller-provided instance of LCG with the contents of the Angel internal $G^{*}$. The propagation through the remainder graph is encapsulated entirely in xaifBooster as a code generation step. Note that the heuristic logic inside reducing_edge_eliminations is no longer as simple as, for instance, the Markowitz criterion because we need to precompute the effect an elimination would have on the edge count considering different combinations of unit/constant and variable edge labels. This precomputation is implemented in edge_elim_effect. Other implementations of scarcity-preserving heuristics can be found in compute_partial_transformation_sequence which includes logic to produce pre- and postrouting steps, and in the _random variants of the above, which include randomized choices and backtracking in heuristics rather than the simple greedy algorithm explained here.

\section{Reversal Schemes}

Section 1 introduces reversal schemes as a means to obtain the $\boldsymbol{J}_{i}$ in reverse order, potentially involving recomputation as a tradeoff for storage. In OpenAD the granularity of choice for making this tradeoff is the subroutine call.

\subsection{Simple Split and Joint Modes}

For a given scope the two extreme ends of the tradeoff are called split mode and joint mode. The former stores (or "tapes") all $\boldsymbol{J}_{i}$ at once; the latter minimizes the storage for 


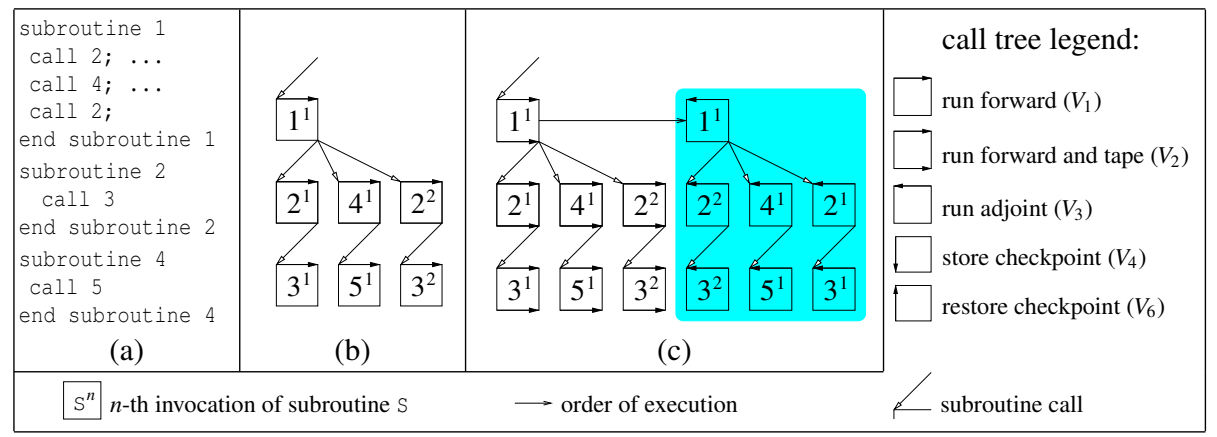

Fig. 5. Example code (a), corresponding call tree (b), split mode (c), and legend.

the $J_{i}$ and the checkpoints. A split mode example is shown in Fig. 5. The adjoint phase, that is, the propagation $\left(\ldots\left(\boldsymbol{W}^{T} \circ \boldsymbol{J}_{s}\right) \circ \ldots \circ \boldsymbol{J}_{1}\right)$, is colored blue. The joint mode is characterized by the fact that we store the $\boldsymbol{J}_{i}$ for each subroutine only immediately before the corresponding adjoint sweep. A joint mode corresponding to Fig. 5(a) is shown in Fig. 6 where the pairs of storing the $\boldsymbol{J}_{i}$ followed by the adjoint are colored green and recomputations are framed in red. Both schemes exhibit a very regular structure

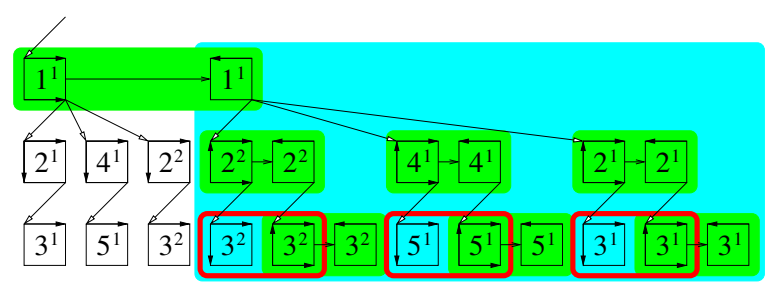

Fig. 6. Joint mode for Fig. 5(a). in which each subroutine executes one of five specific variants $\left(V_{1}-V_{4}, V_{6}\right)$ generated by OpenAD. ${ }^{7}$ Rather than the extremes, in practice a hybrid reversal scheme is used, consisting in part of the split mode for as large a section of the model for which the corresponding $\boldsymbol{J}_{i}$ still fit into memory, while the higher-level parts use some checkpointing scheme and the joint mode. Because this is an application-dependent problem, we do not directly generate the entire reversal scheme in xaifBooster but rather use a template and a postprocessing step to orchestrate the reversal.

\subsection{Template Mechanism}

An OpenAD template is best understood as a sample subroutine with some control flow into which at predefined spots the postprocessor inserts the variants $V_{i}$. While the xaifBooster transformation generates the $V_{i}$, the control structure together with some static state information determines which version $V_{i}$ at any invocation point in the call tree is executed. As examples, the split and joint mode templates are shown in Fig. 7(a) and (b), respectively; the control structure is shown in blue. The numbering of the subroutine variants $\left(V_{1}-V_{4}, V_{6}\right)$ directly reflects the $i d$ number referenced in the

\footnotetext{
7 Other generated variants $V_{5}, V_{7}$ etc. are not of relevant for this paper.
} 


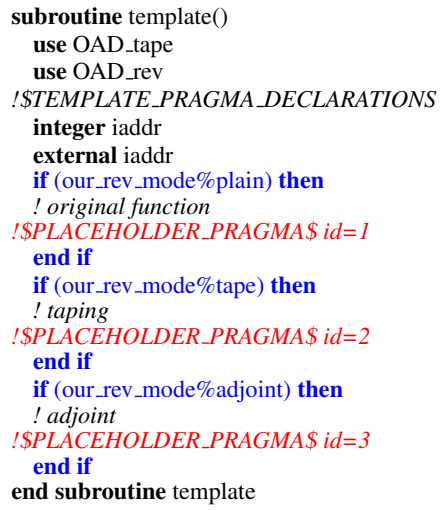

Fig. 7. Split mode template (a); joint mode template (b). The definitions of the OAD_rev* routines can be found in \$OPENADROOT/runTimeSupport/simple/OAD_rev.f90.

! \$PLACEHOLDER_PRAGMA\$ (shown in red). The logic for the split mode is self-evident: the driver for the program sets the global our_rev_mode and invokes the top-level model routine once with our_rev_mode\%tape and once with our_rev_mode\%adjoint set to true. The joint mode logic in the template is more complicated, but one can easily see that the execution of each subroutine variant implies which variant should be executed for its callees. The callee variant is set through calls to the respective $O A D \_r e v^{\star}$ routines (shown in Fig. 7(b) in green); and by consulting the joint scheme figure, one can easily verify the correctness of the template logic. In particular, we see that after storing the $\boldsymbol{J}_{i}$ for the given subroutine (line 20) the mode is set to adjoint (line 21), which then immediately follows (lines 23-27) as expected in the joint mode.

\subsection{Reversal Scheme Using Revolve}

In uniform time-stepping schemes one can assume that all checkpoints are of the same size, as are the storage requirements to produce the $\boldsymbol{J}_{i}$ of a single timestep. This can be used to derive an optimal reversal scheme [19] that minimizes the number of recomputations for a given total $s$ of timesteps and permitted number $p$ of checkpoints. Rather than a strict split or joint mode it indicates for each step, to the top-level time step routine and all its callees, which of the respective variants $V_{i}$ should be executed. A Fortran version of optimal algorithm is available. ${ }^{8}$ Figure 8 (a) shows an example loop

\footnotetext{
${ }^{8}$ See http://mercurial.mcs.anl.gov/ad/RevolveF9X.
} 


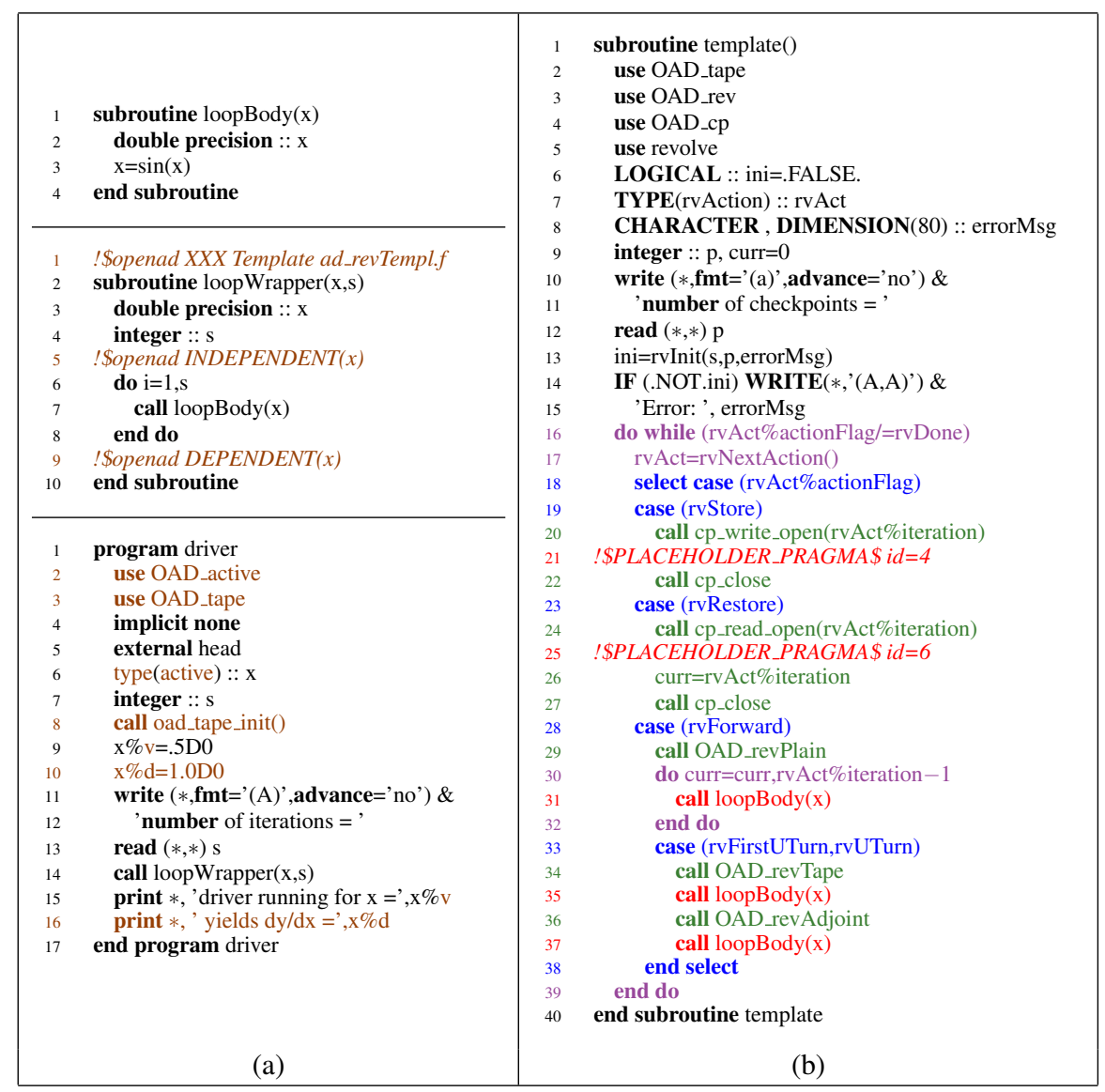

Fig. 8. Time-stepping example loop in loopWrapper with the loop body encapsulated in loopBody and a driver (a); ad_revTempl. $f$ to be applied to loopWrapper (b).

code with adjustments needed to apply OpenAD shown in brown; see also [3], Sec. 1.3. Figure 8(b) shows the template to be applied to loopWrapper and used in conjunction with revolve. The key ingredients of the template are the loop (line 16) replacing the original time-stepping loop (line 6) in loopWrapper. All actions are determined by calling rvNextAction (line 17). We distinguish storing and restoring checkpoints to file by injecting the subroutine variants $V_{4}$ (line 21) and $V_{6}$ (line 25), respectively, computing forward (lines 30-32) up to a step determined by revolve, and doing split adjoint computation (lines 34-37) for rvFirstuTurn and rvUTurn. In the latter the loopBody is directly injected (lines 35,37 ) because in the template in Fig. 8(b) we explicitly replace the entire loop construct of loopWrapper (lines 6-8). Consequently, for the template mechanism it is important to have the time-stepping loop separated in a wrapper, as done in our example. While the above represents the solution to a very regular setup, one can use the same idea to apply new heuristics in cases where the problem is 
combinatorial, for example, when the timesteps (and therefore the associated storage requirements) are not homogeneous. We note that the template mechanism as the entry point to the overall reversal is well insulated from the remainder of the OpenAD tool chain and provides an easy access to experiment with other reversal schemes.

Acknowledgements This work was supported by the Mathematical, Information, and Computational Sciences Division subprogram of the Office of Advanced Scientific Computing Research, Office of Science, U.S. Dept. of Energy under Contract DEAC02-06CH11357.

\section{References}

1. Griewank, A., Walther, A.: Evaluating Derivatives: Principles and Techniques of Algorithmic Differentiation. 2nd edn. Number 105 in Other Titles in Applied Mathematics. SIAM, Philadelphia, PA (2008)

2. OpenAD website: downloads, manual, links. http://www.mcs.anl.gov/openad

3. Utke, J., Naumann, U.: OpenAD/F: User Manual. Technical report, Argonne National Laboratory (2009) latest version available online at http://www.mcs.anl.gov/OpenAD/openad.pdf.

4. Bücker, H.M., Corliss, G.F., Hovland, P.D., Naumann, U., Norris, B., eds.: Automatic Differentiation: Applications, Theory, and Implementations. Volume 50 of Lecture Notes in Computational Science and Engineering. Springer, New York (2005)

5. Bischof, C.H., Bücker, H.M., Hovland, P.D., Naumann, U., Utke, J., eds.: Advances in Automatic Differentiation. Volume 64 of Lecture Notes in Computational Science and Engineering. Springer, Berlin (2008)

6. AD community website: news, tools collection, bibliography. http://www. autodiff.org/

7. Hovland, P., Naumann, U., Walther, A.: Combinatorial problems in automatic differentiation. In Naumann, U., Schenk, O., Simon, H., Toledo, S., eds.: Combinatorial Scientific Computing. Dagstuhl Seminar Proceedings, Dagstuhl, Germany, Schloss Dagstuhl - LeibnizZentrum fuer Informatik, Germany (2009)

8. Naumann, U., Hu, Y.: Optimal vertex elimination in single-expression-use graphs. ACM Transactions on Mathematical Software 35 (2008)

9. Utke, J.: Flattening basic blocks. [4] 121-133

10. Baur, W., Strassen, V.: The complexity of partial derivatives. Theoretical Computer Science 22 (1983) 317-330

11. Naumann, U.: Optimal accumulation of Jacobian matrices by elimination methods on the dual computational graph. Mathematical Programming, Ser. A 99 (2004) 399-421

12. Naumann, U.: Optimal Jacobian accumulation is NP-complete. Math. Prog. 112 (2006) 427-441

13. Griewank, A.: A mathematical view of automatic differentiation. In: Acta Numerica. Volume 12. Cambridge University Press (2003) 321-398

14. Lyons, A., Utke, J.: On the practical exploitation of scarsity. [5] 103-114

15. Naumann, U.: Call tree reversal is NP-complete. [5] 13-22

16. Boost $\mathrm{C}++$ Libraries website: downloads, documentation, news.

http: //www. autodiff.org/

17. AD nested graph elimination library (angel) website: downloads, overview. http://angellib.sourceforge.net 
18. Naumann, U., Gottschling, P.: Simulated annealing for optimal pivot selection in Jacobian accumulation. In Albrecht, A., Steinhöfel, K., eds.: Stochastic Algorithms: Foundations and Applications. Volume 2827 of Lecture Notes in Computer Science., Springer (2003) 83-97

19. Griewank, A., Walther, A.: Algorithm 799: Revolve: An implementation of checkpoint for the reverse or adjoint mode of computational differentiation. ACM Transactions on Mathematical Software 26 (2000) 19-45 Also appeared as Technical University of Dresden, Technical Report IOKOMO-04-1997. The submitted manuscript has been created by UChicago Argonne, LLC, Operator of Argonne National Laboratory ("Argonne"). Argonne, a U.S. Department of Energy Office
of Science laboratory, is operated under Contract No. DE-AC02-06CH1 1357. The U.S. Government retains for itself, and others acting on its behalf, a paid-up, nonexclusive,
irrevocable worldwide license in said article to reproduce, prepare derivative works, distribute copies to the public, and perform publicly and display publicly, by or on behalf of the Government. 